\title{
EFECTO DE CUATRO NIVELES DE SOMBREAMIENTO SOBRE LA CALIDAD DE VARA FLORAL Y CORMO DE LIATRIS (Liatris spicata)
}

\section{Effect of four shading levels on flower stem and corm quality of liatris (Liatris spicata)}

\author{
Flavia Schiappacasse C. ${ }^{\text {* }}$, Gilda Carrasco S. ${ }^{1}$ y Fabiola Carrasco C. ${ }^{1,2}$
}

\begin{abstract}
A B S T R A C T
Liatris spicata (L.) Willd. was introduced to Chile as a cut flower crop more than a decade ago. It is commonly field-planted in consecutive plantings during spring and summer. The propagation structure is a corm that is reused in subsequent plantings. Other field crops are shaded to reduce the temperature, but in liatris, the effect of shading under our conditions on both cut flowers and corms is unknown. The present study assessed the cut flower quality of $L$. spicata cultivated in the VII Region ( $35^{\circ} 33^{\prime} \mathrm{S}$ lat, $71^{\circ} 13^{\prime} \mathrm{W}$ long), Chile, utilizing nets providing 35, 50, 65 and $80 \%$ shade, plus a control treatment without shade. As well, the weight of the corms was recorded one, four and eight weeks after flower harvest. The effect of shade on flower quality was evident at harvest time; the plants that grew at full sun had longer spikes and greater stem diameters. The weight of corms at the third evaluation was greatest in the control treatment, and at the first evaluation these values were lower than in the last evaluation. As the shade negatively affected both flower quality and corm weight, it was concluded that shading nets should not be recommended under this experimental conditions.
\end{abstract}

Key words: Liatris spicata, liatris, cut flower, bulb, corm, light level.

\section{R E S U M E N}

El cultivo de Liatris spicata (L.) Willd. para flor cortada fue introducido en Chile hace más de una década. Se suele plantar en forma escalonada al aire libre en primavera y verano. Su estructura de propagación corresponde a un cormo, el cual se reutiliza en la siguiente plantación. Otros cultivos al aire libre se sombrean para reducir la temperatura, pero en liatris se desconoce el efecto del sombreamiento en nuestras condiciones, tanto para la vara floral como para el cormo. El presente estudio evaluó la calidad de las varas florales de L. spicata, cultivadas en la VII Región ( $35^{\circ} 33^{\prime}$ lat. Sur, $71^{\circ} 13^{\prime}$ long. Oeste) bajo diferentes niveles de sombra, utilizando mallas de 35; 50; 65 y $80 \%$ de sombra y un testigo sin sombra. Además se midió el peso de los cormos al cabo de una, cuatro y ocho semanas después de la cosecha de flores. El efecto de la sombra sobre la calidad de las varas se manifestó al momento de cosecha, encontrándose una mayor longitud de espiga y diámetro de varas en las plantas que crecieron a pleno sol. El peso de cormos en la tercera evaluación fue superior en el tratamiento testigo, y en la primera evaluación los valores fueron inferiores a los de la última evaluación. La sombra afectó negativamente la calidad de flores y el peso del cormo, por lo que se concluye que en las condiciones del presente experimento no se recomienda el uso de mallas sombreadoras para su cultivo.

Palabras clave: Liatris spicata, liatris, flor cortada, bulbo, cormo, nivel de luz.

\footnotetext{
${ }^{1}$ Universidad de Talca, Facultad de Ciencias Agrarias, Casilla 747, Talca, Chile.

E-mail: fschiap@utalca.cl *Autor para correspondencia.

${ }^{2}$ Dirección particular: $5^{1 / 2}$ Norte 3074 , Talca.

Recibido: 12 de abril de 2005. Aceptado: 24 de agosto de 2005.
} 


\section{INTRODUCCIÓN}

El cultivo de liatris, Liatris spicata (L.) Willd., para flor cortada fue introducido en Chile hace más de una década y ha estado entre las principales especies exportadas durante varios años. Pertenece a la familia Asteraceae. El género es originario de Norteamérica. Su inflorescencia es una espiga de florecillas sésiles de color púrpura. Su estructura de propagación corresponde a un cormo, el cual comúnmente se planta en forma escalonada al aire libre desde inicios de primavera hasta el verano, para cosechar las flores desde fines de primavera hasta el otoño. Los cormos necesitan ser almacenados previamente en frío, entre 3 y $5^{\circ} \mathrm{C}$ al menos por ocho semanas para lograr una floración óptima (Waithaka y Wanjao, 1982). Se describe como una planta de día largo facultativa; las condiciones de día largo promueven su floración, pero ésta ocurre bajo cualquier fotoperíodo (Moe, 1993). Por ende, su cultivo es posible todo el año. Para cultivos estivales, el Centro Internacional de Bulbos de Flor (1992) recomienda la utilización de mallas de alrededor de $45 \%$ de sombra en regiones de alta luminosidad. Sin embargo, en nuestras condiciones se desconoce el efecto de la sombra. Un efecto buscado al utilizar sombreamiento es la reducción de temperaturas, y otro efecto es la elongación de los internudos, y, por consiguiente, una mayor longitud de vara floral. Armitage (1991) comprobó que el sombreamiento en Anemone coronaria 'De Caen' produjo tallos más largos, sin afectar el rendimiento de flores.

Es importante destacar que en nuestro país se reutilizan los cormos de liatris, por lo tanto, es necesario conocer el efecto del sombreamiento sobre ellos, así como también determinar el momento de sacarlos del suelo después de la cosecha de las flores.

El objetivo general del presente estudio fue evaluar la calidad de la flor cortada y el cormo de liatris, en plantas cultivadas bajo distintos niveles de radiación solar, para lo cual se establecieron los siguientes objetivos específicos: determinar el nivel de sombreamiento más adecuado para obtener una vara floral de mejor calidad, en cuanto a longitud de la vara, longitud de la espiga y diámetro del tallo, comparado con un testigo sin sombra; determinar cómo afectan los niveles de sombreamiento al peso de los cormos, y determinar cuál es el momento más adecuado para cosecharlos.

\section{MATERIALES Y MÉTODOS}

El estudio se realizó en un predio ubicado en San Clemente $\left(35^{\circ} 17^{\prime}\right.$ lat. Sur, $71^{\circ} 17^{\prime}$ long. Oeste) a 20 $\mathrm{km}$ al oriente de Talca, durante los meses de octubre de 1996 a marzo de 1997. El suelo corresponde a la serie Talca (Ultic Haploxeralfs) (CIREN, 1997).

Los cormos de liatris se importaron originalmente desde Israel, y habían sido cultivados en el predio por varias temporadas. Medían 3 a $5 \mathrm{~cm}$ de circunferencia. Previo a la plantación, se desinfectaron mediante inmersión de 20 minutos en una solución de captan 1,6 g i.a. $\mathrm{L}^{-1}$ (Captan $80 \mathrm{WP}$ ), benomil 0,3 g i.a. $\mathrm{L}^{-1}$ (Benlate), sulfato de estreptomicina + clorhidrato de oxitetraciclina $\left(0,2 \mathrm{~g}\right.$ i.a. $\mathrm{L}^{-1}+0,03 \mathrm{~g}$ i.a. $\left.\mathrm{L}^{-1}\right)$ (Streptoplus), carboxina + thiram $(2 \mathrm{~g}$ i.a. $\mathrm{L}^{-1}+2$ g i.a. $\left.\mathrm{L}^{-1}\right)$ (Vitavax) y carbofurano $0,4 \mathrm{~g}$ i.a. $\mathrm{L}^{-1}$ (Furadan 4F).

La plantación se realizó el 15 de octubre de 1996 al aire libre y bajo mallas sombreadoras negras de distinto grado de sombra. La preparación del suelo incluyó el uso de arado con cincel, rastra y rotovator para lograr un adecuado mullimiento en los primeros 20-25 cm del suelo. Se confeccionaron platabandas de $1 \mathrm{~m}$ de ancho.

El riego se efectuó mediante cintas de goteo (cuatro por platabanda), con una frecuencia de riego de cuatro días al inicio de la temporada y un día en pleno verano. El tiempo de riego fluctuó entre $60 \mathrm{y}$ 120 min. Se utilizó fertirrigación. Los principales fertilizantes fueron Ultrasol de crecimiento (25-10$10+1 \mathrm{MgO}+1 \mathrm{~S}+$ microelementos), Ultrasol de producción (13-6-40) (ambos de SOQUIMICH, Chile); Nitrato de magnesio ( $11 \%$ de $\mathrm{N}$ y $15 \%$ de $\mathrm{Mg})$ y Nitrato de calcio $(15,5 \%$ de $\mathrm{N}$ y $19 \%$ de $\mathrm{Ca})$.

El control fitosanitario fue principalmente preventivo para controlar Rhizoctonia sp., Botrytis sp. y hongos oomycetes. El control de malezas durante el cultivo fue manual y se realizó cada dos semanas.

La plantación se realizó en una platabanda de $60 \mathrm{~m}$ de largo. Se dividió en parcelas de $3 \mathrm{~m}$ de largo, disponiéndose en forma aleatoria sobre cada una las distintas mallas sombreadoras negras (CORESA) de 35, 50, 60 y $80 \%$ de sombra y el control sin sombra, quedando los cinco tratamientos con cuatro repeticiones cada uno. Las mallas se dis- 
pusieron a 2,5 $\mathrm{m}$ de alto, y tenían una dimensión de $3 \mathrm{~m}$ largo por $8 \mathrm{~m}$ de ancho, alcanzando a cubrir las platabandas y sus costados. Se utilizó una densidad de plantación de 120 cormos $\mathrm{m}^{-2}$. La cosecha de flores de liatris comenzó a los 80 días después de la plantación, y se efectuó dejando 10 a $15 \mathrm{~cm}$ de tallo sobre la superficie del suelo.

Los tratamientos evaluados fueron: $0 \%$ sombra (control); $35 \%$ sombra; $50 \%$ sombra; $65 \%$ sombra y $80 \%$ sombra. Para determinar el mejor nivel de sombreamiento sobre la calidad de las varas florales, dentro de cada repetición de cada tratamiento se marcaron cinco plantas del centro de la parcela, evaluándose las siguientes variables: altura de planta, se registró semanalmente, y consistió en la longitud del tallo medida desde la superficie del suelo hasta el ápice de crecimiento; longitud de vara al momento de cosecha, medida desde la superficie del suelo hasta el ápice del botón floral superior; diámetro de tallo, medido con pie de metro al momento de la cosecha en el sector medio del tallo; longitud de la espiga, medida al momento de la cosecha; fecha de madurez de cosecha, correspondió a la fecha en que el 50\% de las flores de la parcela habían sido cosechadas.

Para comparar la calidad de los cormos en los distintos tratamientos de sombra, y determinar el momento más adecuado para su extracción desde el suelo, se consideró el peso fresco de los cormos, para lo cual se tomaron 10 cormos de cada tratamiento y repetición en tres fechas distintas: 1, 4 y 8 semanas después de la cosecha de flores, y se pesaron en una balanza.

Para efecto de comparar la intensidad de luz bajo las distintas mallas sombreadoras, se registró la radiación fotosintéticamente activa (PAR) en una oportunidad, a los 62 días después de la plantación, en un día despejado, en cada repetición de cada tratamiento a la altura de los ápices de las plantas. Además, se midió la temperatura ambiental a 1,5 $\mathrm{m}$ de altura y a nivel de suelo.

La respuesta de las variables se analizó con un diseño completamente al azar. Los resultados obtenidos se sometieron a análisis estadístico, realizándose análisis de varianza y, cuando correspondió, se aplicó la prueba de comparaciones múltiples de Duncan $(\mathrm{P} \leq 0,05)$.

\section{RESULTADOS Y DISCUSIÓN}

No se observaron diferencias significativas en los valores de longitud de vara entre tratamientos en las primeras evaluaciones, pero desde la evaluación realizada el 6 de enero hubo diferencias ( $p \leq 0,05)$, aunque sólo entre el tratamiento control y de $65 \%$ de sombra (Cuadro 1); los valores del resto de los tratamientos no fueron distintos a los de estos dos tratamientos. Según Armitage (1991), tubérculos de Anemone coronaria 'De Caen' cultivados a pleno sol, $55 \%$ y $67 \%$ de sombra, produjeron plantas más altas bajo $67 \%$ de sombra, seguidas por las plantas bajo $55 \%$ de sombra, sin variaciones en el rendimiento de flores. El mismo autor (1993) recomienda el uso de sombra en condiciones de alta luminosidad en cultivos de Amaranthus caudatus, Anthirrinum majus, Callistephus chinensis, Caryopteris incana, Nigella damascena, Astilbe arendsii, Echinops bannaticus y Zantedeschia spp., pues en tales especies se producen varas de mayor longitud que a pleno sol, sin afectar negativamente la apariencia de la flor y el rendimiento de flores. No menciona el efecto de la sombra en liatris. Cabe mencionar que el mercado no valora mejor las varas de más de $100 \mathrm{~cm}$, además tal longitud dificulta y encarece el embalaje.

Cuadro 1. Altura de plantas de liatris $(\mathrm{cm})$ durante el período de crecimiento. Plantación realizada el 15 de octubre. Talca, Chile. 1996-1997.

Table 1. Liatris plant height $(\mathrm{cm})$ during the growing period. Planted on October $15^{\text {th }}$. Talca, Chile. 1996-1997.

\begin{tabular}{lccccccc}
\hline Tratamiento & 3 Dic. & 10 Dic. & 17 Dic. & 28 Dic. & 6 Ene. & 11 Ene. & 18 Ene. \\
\hline Control & $42,29 \mathrm{a}$ & $60,14 \mathrm{a}$ & $86,82 \mathrm{a}$ & $107,22 \mathrm{a}$ & $115,22 \mathrm{~b}$ & $116,08 \mathrm{~b}$ & $118,08 \mathrm{~b}$ \\
$35 \%$ sombra & $41,36 \mathrm{a}$ & $58,39 \mathrm{a}$ & $90,39 \mathrm{a}$ & $109,18 \mathrm{a}$ & $120,75 \mathrm{ab}$ & $122,46 \mathrm{ab}$ & $123,39 \mathrm{ab}$ \\
$50 \%$ sombra & $44,79 \mathrm{a}$ & $62,68 \mathrm{a}$ & $94,21 \mathrm{a}$ & $112,56 \mathrm{a}$ & $123,32 \mathrm{ab}$ & $124,32 \mathrm{ab}$ & $125,70 \mathrm{ab}$ \\
$65 \%$ sombra & $46,11 \mathrm{a}$ & $62,36 \mathrm{a}$ & $96,50 \mathrm{a}$ & $113,80 \mathrm{a}$ & $127,00 \mathrm{a}$ & $129,75 \mathrm{a}$ & $131,22 \mathrm{a}$ \\
$80 \%$ sombra & $43,86 \mathrm{a}$ & $57,36 \mathrm{a}$ & $89,46 \mathrm{a}$ & $119,43 \mathrm{a}$ & $119,43 \mathrm{ab}$ & $122,00 \mathrm{ab}$ & $124,37 \mathrm{ab}$ \\
\hline
\end{tabular}

Valores dentro de una columna seguidos por una misma letra no difieren estadísticamente según prueba de Duncan $(\mathrm{p} \leq 0,05)$. 
En cuanto a la longitud de espiga y diámetro de vara de liatris, se observó que al aumentar el grado de sombreamiento de las plantas, se redujeron la longitud de la espiga y el diámetro del tallo, lo que afectó la firmeza de la vara (Cuadro 2). El tratamiento de $80 \%$ de sombra produjo los valores más bajos en estos parámetros. Si bien es cierto que se obtuvieron varas más largas con $65 \%$ de sombra, éstas fueron más débiles y produjeron espigas más cortas que las del tratamiento control.

En definitiva, la calidad de vara de las plantas de liatris cultivadas a pleno sol fue mejor que la de plantas sombreadas. La altura de plantas del tratamiento control permitió la cosecha de varas suficientemente largas para ser comercializadas, teniendo en cuenta que se dejan al menos $10 \mathrm{~cm}$ de altura de tallo en el suelo al cosechar, y en diámetro de vara y longitud de espiga se obtuvieron valores que estuvieron entre los más altos. Esto indica que no es recomendable el uso de mallas sombreadoras en plantaciones realizadas en octubre, y tal vez suceda lo mismo en plantaciones posteriores a esa fecha.

El efecto del sombreamiento sobre la fecha de madurez de cosecha (Cuadro 3) indicó que bajo las mallas sombreadoras, la maduración de las varas florales fue más lenta, atrasándose aproximadamente en 11 días para las plantas que crecieron bajo $80 \%$ de sombra en comparación con el tratamiento control. Este retraso en la maduración podría atribuirse a la menor temperatura existente bajo la malla sombreadora, que pudo atrasar el crecimiento y desarrollo de las plantas. Este efecto de las bajas temperaturas de crecimiento sobre el retraso en la floración en liatris fue observado antes por Espinosa et al. (1991).

Cuadro 2. Diámetro de vara $(\mathrm{mm})$ y longitud de espiga (cm) al momento de la cosecha de flores de liatris.

Table 2. Flower stem diameter $(\mathrm{cm})$ and spike length (cm) at the time of liatris flower harvest.

\begin{tabular}{lcc}
\hline Tratamiento & Diámetro de vara & Longitud de espiga \\
\hline Control & $6,28 \mathrm{a}$ & $21,71 \mathrm{a}$ \\
$35 \%$ sombra & $5,40 \mathrm{~b}$ & $19,54 \mathrm{ab}$ \\
$50 \%$ sombra & $5,61 \mathrm{ab}$ & $18,36 \mathrm{~b}$ \\
$65 \%$ sombra & $5,61 \mathrm{ab}$ & $19,96 \mathrm{~b}$ \\
$80 \%$ sombra & $4,75 \mathrm{c}$ & $15,57 \mathrm{c}$ \\
\hline
\end{tabular}

Valores dentro de una columna seguidos por una misma letra no difieren estadísticamente según prueba de Duncan $(\mathrm{p} \leq 0,05)$.
Cuadro 3. Días a madurez de cosecha de flores de liatris bajo distintos niveles de sombra (días después de la plantación) ${ }^{1}$.

Table 3. Days of harvest maturity of flowers liatris under different shading levels (days after planting) ${ }^{1}$.

\begin{tabular}{lc}
\hline Tratamiento & Días a cosecha \\
\hline Control & 88 \\
$35 \%$ sombra & 95 \\
$50 \%$ sombra & 96 \\
$65 \%$ sombra & 97 \\
$80 \%$ sombra & 99 \\
\hline
\end{tabular}

${ }^{1}$ Sin análisis estadístico.

Después de la cosecha de las flores, el cormo sigue creciendo (Cuadro 4), aparentemente a expensas de la roseta que se formó alrededor de la base del tallo, así como del tallo que fue cortado. Al comparar entre tratamientos, en la primera y en la tercera evaluación los cormos del tratamiento testigo tuvieron un mayor peso que los de los otros tratamientos.

En cuanto al análisis para determinar la fecha adecuada para la cosecha de los cormos, sólo puede decirse que éstos se deben cosechar después de la primera semana desde la cosecha de flores, ya que hubo aumento de peso en las siguientes evaluaciones. Sin embargo, no hubo diferencias estadísticas entre los valores de la $4^{\mathrm{a}}$ y $8^{\mathrm{a}}$ semanas, y no se sabe si habría aumento de peso al dejar los cormos más tiempo en el suelo porque no se realizaron más evaluaciones.

En un estudio realizado en Sandersonia aurantiaca (Hook.) por Clark y Reyngoud (1997), en que también se fueron cosechando los órganos subte-

Cuadro 4. Peso de cormos de liatris después de la cosecha de flores.

Table 4. Liatris corm weight after flower harvest.

\begin{tabular}{lccc}
\hline Tratamiento & $\mathbf{1}^{\text {a }}$ semana & $\mathbf{4}^{\text {a }}$ semana & $\mathbf{8}^{\text {a }}$ semana \\
\hline Control & $17,11 \mathrm{aB}$ & $20,60 \mathrm{aA}$ & $22,70 \mathrm{aA}$ \\
$35 \%$ sombra & $14,34 \mathrm{bB}$ & $20,49 \mathrm{aA}$ & $17,83 \mathrm{bA}$ \\
$50 \%$ sombra & $13,19 \mathrm{bB}$ & $18,94 \mathrm{aA}$ & $19,56 \mathrm{bA}$ \\
$65 \%$ sombra & $13,62 \mathrm{bB}$ & $18,29 \mathrm{aA}$ & $18,70 \mathrm{bA}$ \\
$80 \%$ sombra & $13,98 \mathrm{bB}$ & $18,02 \mathrm{aA}$ & $19,16 \mathrm{bA}$ \\
\hline
\end{tabular}

Valores dentro de una columna seguidos por una misma letra minúscula no difieren estadísticamente; valores dentro de una fila seguidos por una misma letra mayúscula no difieren estadísticamente, según prueba de Duncan $(\mathrm{p} \leq 0,05)$. 
rráneos, en este caso tubérculos, posterior a la floración en distintas fechas, éstos aumentaron de tamaño hacia las últimas fechas de evaluación (5 y 9 semanas después de floración), y los valores fueron similares entre sí.

Los valores medios de PAR fueron de 1.750, 1.000, 773,481 y $325 \mu \mathrm{mol} \mathrm{m}{ }^{-2} \mathrm{~s}^{-1}$ para los tratamientos control, $35,50,65$ y $80 \%$ de sombra, respectivamente. Los valores de temperatura registrados en el tratamiento testigo fueron superiores a los del resto de los tratamientos. La temperatura a $1,5 \mathrm{~m}$ en el tratamiento testigo fue $32^{\circ} \mathrm{C}, 8^{\circ} \mathrm{C}$ superior a la del tratamiento de $80 \%$ de sombra, mientras a nivel de suelo en el tratamiento testigo la tempera- tura fue $24^{\circ} \mathrm{C}$, y entre ambos tratamientos la diferencia fue de $5^{\circ} \mathrm{C}$.

\section{CONCLUSIONES}

La sombra afectó negativamente la calidad de flores de liatris, disminuyendo la longitud de espiga y el diámetro de vara, por lo que no se recomienda el uso de mallas sombreadoras para su cultivo.

El crecimiento de cormos también fue afectado negativamente en los tratamientos con mallas sombreadoras. Los cormos cultivados sin sombra deberían cosecharse después de la primera semana desde la cosecha de las flores.

\section{LITERATURA CITADA}

Armitage, A. M. 1991. Shade affects yield and stem length of field-grown cut-flower species. HortScience 26:1174-1176.

Armitage, A. M. 1993. Specialty cut flowers. 372 p. Varsity Press, Inc./Timber Press, Inc., Portland, Oregon, USA.

Centro Internacional de Bulbos de Flor. 1992. La liatris como flor cortada. Parklaan 5, P.O. Box 172, 2180 AD, Hillegom, Holanda.

CIREN. 1997. Descripciones de suelos, materiales y símbolos. Estudio Agrológico VII Región. Tomo 1. Publicación CIREN $N^{\circ}$ 117. Centro de Investigación de Recursos Naturales (CIREN), Santiago, Chile.
Clark, G.E., and J.L. Reyngoud. 1997. Effects of production methods on Sandersonia cut stem quality and tuber size. Acta Hortic. 430:731-735.

Espinosa, I., W. Healy, and M. Roh. 1991. The role of temperature and photoperiod on Liatris spicata shoot development. J. Am. Soc.Hortic. Sci. 116:27-29.

Moe, R. 1993. Liatris. p. 381-390. In De Hertogh, A. and M. Le Nard (eds.). The physiology of flower bulbs. Elsevier Science Publishers B.V., Amsterdam, The Netherlands.

Waithaka, K., and L.W. Wanjao. 1982. The effect of duration of cold treatment on growth and flowering of Liatris. Sci. Hortic.18:153-158. 